\title{
The Hamburg/SAO survey for emission-line galaxies
}

\section{The fifth list of 161 galaxies}

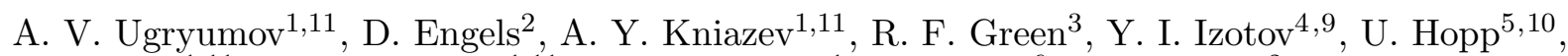 \\ S. A. Pustilnik ${ }^{1,11}$, A. G. Pramsky ${ }^{1,11}$, T. F. Kniazeva ${ }^{1}$, N. Brosch ${ }^{6}$, H.-J. Hagen ${ }^{2}$, V. A. Lipovetsky ${ }^{1, \star}$,
} J. Masegosa ${ }^{7}$, I. Márquez ${ }^{7}$, and J.-M. Martin ${ }^{8}$

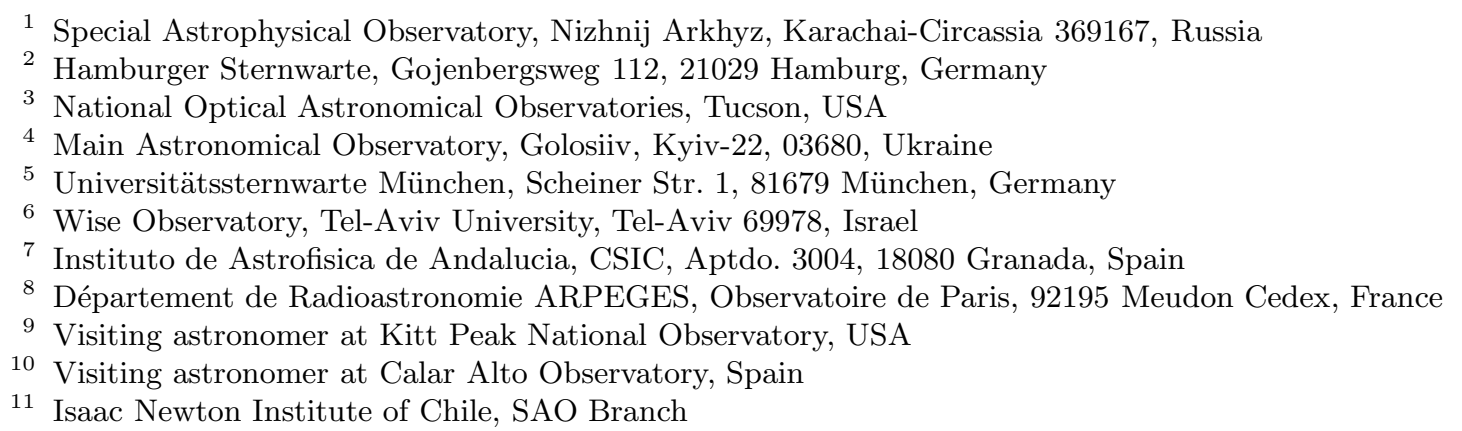

Received 3 April $2001 /$ Accepted 31 May 2001

\begin{abstract}
We present the fifth list with results ${ }^{\star \star}$ of the Hamburg/SAO Survey for Emission-Line Galaxies (HSS therein, SAO - Special Astrophysical Observatory, Russia). The list is a result of follow-up spectroscopy conducted with the $2.2 \mathrm{~m}$ CAHA and $4 \mathrm{~m}$ Kitt Peak telescopes in 1999. The data of this snap-shot spectroscopy survey confirmed 166 emission-line objects out of 209 observed candidates and allowed their quantitative spectral classification and redshift determination. We could classify 98 emission-line objects as BCG/Hit galaxies or probable BCGs, 5 - as QSOs, 3 - as Seyfert galaxies, 2 - as super-associations in subluminous spiral galaxies. 25 low-excitation objects were classified as starburst nuclei (SBN), 24 as dwarf amorphous nuclei starburst galaxies (DANS) and 3 as LINERs. Due to low signal-to-noise ratio we could not classify 6 ELGs. Furthermore, for another 4 galaxies we did not detect any significant emission lines. For 131 emission-line galaxies, the redshifts and/or line intensities are determined for the first time. For the remaining 30 previously known ELGs we give either improved data of the line intensities or some independent measurements.
\end{abstract}

Key words. surveys - galaxies: fundamental parameters - galaxies: distances and redshifts - galaxies: starburst quasars: general

\section{Introduction}

The problem of creating large, homogeneous and deep samples of actively star-forming low-mass galaxies is very important for several applications in studies of galaxy evolution and spatial distribution. Several earlier projects, like the Second Byurakan Survey (SBS) (Markarian et al. 1983; Stepanian 1994), the University of Michigan (UM)

Send offprint requests to: A. V. Ugryumov

e-mail: and@sao.ru

* Deceased 1996 September 22.

** Tables 3 to 7 are only available in electronic form at the CDS via anonymous ftp to cdsarc.u-strasbg.fr

$(130.79 .128 .5)$ or via

http://cdsweb.u-strasbg.fr/cgi-bin/qcat?J/A+A/374/907

Figures A1 to A17 are only available in electronic form at http://www.edpsciences.org survey (e.g., Salzer et al. 1989), and the Case survey (Pesch et al. 1995; Salzer et al. 1995; Ugryumov et al. 1998), as well as some others, identified on objective prism plates many hundreds of emission-line galaxies. The Hamburg/SAO survey (HSS) is intended to create a new very large homogeneous sample of such galaxies in the region of the Northern sky with an area of some 1700 square degrees. The basic outline of the HSS and first results are described in Paper I (Ugryumov et al. 1999), while additional results from follow-up spectroscopy are given in Papers II, III and IV (Pustilnik et al. 1999; Hopp et al. 2000; Kniazev et al. 2001a). In this paper we present the results of follow-up spectroscopy of another 209 objects selected on the Hamburg Quasar Survey (HQS) prism spectral plates as ELG candidates.

The article is organized as follows. In Sect. 2 we give the details of the spectroscopic observations and of the 
Table 1. Summary of the samples observed and breakdown of the classifications after follow-up spectroscopy.

\begin{tabular}{|c|c|c|c|c|c|c|c|c|}
\hline \multicolumn{2}{|c|}{ Candidate Sample } & \multirow{2}{*}{$\begin{array}{c}N \\
141\end{array}$} & \multirow{2}{*}{$\begin{array}{c}\mathrm{BCG} \\
\& \\
\text { BCG? } \\
64\end{array}$} & \multirow{2}{*}{$\begin{array}{l}\text { Other } \\
\text { ELGs } \\
\text { \& SA } \\
43\end{array}$} & \multirow{2}{*}{$\begin{array}{c}\text { QSO } \\
5\end{array}$} & \multirow{2}{*}{$\begin{array}{c}\begin{array}{c}\text { Galaxies } \\
\text { without } \\
\text { emission }\end{array} \\
3\end{array}$} & \multirow{2}{*}{$\begin{array}{c}\text { Stars } \\
\\
18\end{array}$} & \multirow{2}{*}{$\begin{array}{c}\text { Not } \\
\text { Classified } \\
8\end{array}$} \\
\hline First priority & new & & & & & & & \\
\hline & already known & 28 & 21 & 7 & - & - & - & - \\
\hline & total & 169 & 85 & 50 & 5 & 3 & 18 & 8 \\
\hline \multirow[t]{2}{*}{ Random sample } & new & 16 & 3 & 1 & - & 1 & 2 & 9 \\
\hline & already known & 1 & - & 1 & - & - & - & - \\
\hline \multirow{2}{*}{ APM selected sample } & new & 22 & 10 & 10 & - & - & 1 & 1 \\
\hline & already known & 1 & - & 1 & - & - & - & - \\
\hline Second priority & total & 40 & 13 & 13 & - & 1 & 3 & 10 \\
\hline \multicolumn{2}{|c|}{ Objects presented in this paper } & 209 & 98 & 63 & 5 & 4 & 21 & 18 \\
\hline
\end{tabular}

Table 2. Journal of observations.

\begin{tabular}{|c|c|c|c|c|c|c|}
\hline Date & Telescope & $\begin{array}{c}\text { Instrument } \\
\text { (3) }\end{array}$ & $\begin{array}{l}\text { Grating, } \\
\text { grism } \\
(4)\end{array}$ & $\begin{array}{c}\text { Wavelength } \\
\text { range }[\AA] \\
(5)\end{array}$ & $\begin{array}{c}\text { Dispersion } \\
{[\AA / \text { pixel }]} \\
(6)\end{array}$ & $\begin{array}{c}\text { Observed } \\
\text { number } \\
(7)\end{array}$ \\
\hline 09.06-18.06.1999 & $2.2 \mathrm{~m} \mathrm{CAHA}$ & CAFOS & G-200 & $3700-9500$ & 4.5 & 117 \\
\hline - & - & - & B-200 & $3500-7400$ & 4.7 & $18^{*}$ \\
\hline 17.06-20.06.1999 & $4 \mathrm{~m} \mathrm{KPNO}$ & R.C.Sp. & KPC-10A & $3700-8300$ & 2.8 & 46 \\
\hline 08.12-11.12.1999 & $2.2 \mathrm{~m} \mathrm{CAHA}$ & CAFOS & G-200 & $3700-9500$ & 9.0 & 46 \\
\hline
\end{tabular}

* - Objects reobserved.

data reduction. In Sect. 3 the results of the observations are presented in several tables. In Sect. 4 we briefly discuss the new data and summarize the current state of the Hamburg/SAO survey. Throughout this paper a Hubble constant $H_{0}=75 \mathrm{~km} \mathrm{~s}^{-1} \mathrm{Mpc}^{-1}$ is used.

\section{Spectral observations and data reduction}

\subsection{Observations}

The results presented here were obtained in a snap-shot observing mode during two runs with the Calar Alto $2.2 \mathrm{~m}$ and one run with the KPNO $4 \mathrm{~m}$ telescopes (see Table 2).

\subsection{Observations with the KPNO $4 m$ telescope}

The observations were carried out with the RitcheyChretien Spectrograph attached to a Tektronix $2 \mathrm{~K} \times 2 \mathrm{~K}$ CCD detector. We used a $2^{\prime \prime} \times 205^{\prime \prime}$ slit with a KPC-10A grating (316 grooves $\mathrm{mm}^{-1}$ ) in its first order, and a GG 375 order separation filter cutting off second-order contamination for wavelengths blueward of $7400 \AA$. This instrumental setup allows a spatial scale along the slit of $0^{\prime \prime} 69 \mathrm{pixel}^{-1}$, a scale perpendicular to the slit of $2.7 \AA$ pixel $^{-1}$, a spectral range of $3700-8300 \AA$ and a spectral resolution of $\sim 7 \AA(F W H M)$. Short exposures (3-5 min.) were used in order to detect strong emission lines to allow measurement of redshifts and a crude classification. No orientation of the slit along the parallactic angle was done because of the snap-shot observing mode. Reference spectra of an Ar-Ne-He lamp were recorded to provide wavelength calibration. Spectrophotometric standard stars from Oke (1990) and Bohlin (1996) were observed at the beginning and at the end of each night for flux calibration. The dome flats, bias, dark and twilight sky frames were accumulated each night. The weather conditions were photometric, with seeing variations between 2 .! 5 and $3^{\prime \prime}$ (FWHM).

\subsection{Calar Alto $2.2 \mathrm{~m}$ telescope observations}

Follow-up spectroscopy with the CAHA $2.2 \mathrm{~m}$ telescope was carried out during two runs (June and December, 1999, see Table 2), using the Calar Alto Faint Object Spectrograph (CAFOS) and Cassegrain focal reducer. During these runs a long slit of $300^{\prime \prime} \times\left(2^{\prime \prime}-3^{\prime \prime}\right)$ and a G-200 grism (187 $\AA \mathrm{mm}^{-1}$, first order) were used. The B-200 grism (185 $\AA \mathrm{mm}^{-1}$, first order) was also used to reobserve 18 objects in order to improve the [OII] $\lambda 3727 \AA$ value. There were no order separation filters applied. The spatial scale along the slit was $0^{\prime \prime} 53$ pixel $^{-1}$. A SITE 15 $2 \mathrm{~K} \times 2 \mathrm{~K}$ CCD was operated in a $2 \times 1$ binned mode during the December run (binning only along the dispersion direction), while in the June run there was no binning applied. The wavelength coverages were $\lambda 3700-\lambda 9500 \AA$ with maximum sensivity at $\sim 6000 \AA$ for the G-200 grism and $\lambda 3500-\lambda 7400 \AA$ with maximum sensivity at $\sim 4000 \AA$ for the B-200 grism. The spectral resolution was $\sim 10 \AA$ $(F W H M)$ in the June run and due to CCD binning 
$\sim 20 \AA(F W H M)$ in the December run. The slit orientation was not aligned with the parallactic angle because of the snap-shot observing mode. The exposure times varied within 2-15 min depending on the object brightness. The observations were complemented by standard star flux measurements (Oke 1990; Bohlin 1996), reference spectra (Hg-Cd lamp) for wavelength calibration, dome flat, bias and dark frames. Most of the time the weather conditions were photometric with a seeing $\approx 1.5^{\prime \prime}(F W H M)$. Only during two nights the weather conditions were variable with a seeing of $3^{\prime \prime}-4^{\prime \prime}(F W H M)$. The measurements of these nights are marked by "*" in Table 4 .

\subsection{Data reduction}

Reduction of the CAHA and KPNO spectral data was performed at the SAO using the standard reduction systems MIDAS $^{1}$ and IRAF ${ }^{2}$.

The MIDAS command FILTER/COSMIC was found to be a quite successful way to remove automatically all cosmic ray hits from the images. After that we applied the IRAF package CCDRED for bad pixel removal, trimming, bias-dark subtraction, slit profile and flat-field corrections.

To do accurate wavelength calibration, correction for distortion and tilt for each frame, sky substraction and correction for atmospheric extinction, the IRAF package LONGSLIT was used with invoking the IDENTIFY, REIDENTIFY, FITCOORD, TRANSFORM, BACKGROUND and EXTINCTION tasks.

To obtain an instrumental response function from observed spectrophotometric flux standards, the APSUM procedure from the APEXTRACT package was used first to extract apertures of standard stars. Then the sensitivity curve determined by the STANDARD and SENSFUNC procedures was applied by the CALIBRATE task to perform flux calibration for all object images. Finally the APSUM task was used to extract one-dimensional spectra from the flux calibrated images. In case that more than one exposure was obtained with the same setup for an object, the extracted spectra were co-added and a mean vector was calculated. In case of several observations with different setups (telescopes or grisms) for the same object, the data were reduced and measured independently and the more reliable values were taken.

To speed-up and facilitate the line measurements we employed dedicated command files created at the SAO using the FIT context and MIDAS command language. The procedures for the measurement of line parameters and redshifts applied were described in detail in Papers III and IV.

\footnotetext{
${ }^{1}$ MIDAS is an acronym for the European Southern Observatory package - Munich Image Data Analysis System.

${ }^{2}$ IRAF is distributed by National Optical Astronomical Observatories, which is operated by the Association of Universities for Research in Astronomy, Inc., under cooperative agreement with the National Science Foundation.
}

\section{Results of follow-up spectroscopy}

In Table 1 we present the results of the observations. The 209 candidates were selected from our first and second priority samples introduced in Paper IV.

Of 169 first priority candidates, 141 objects appeared in our list as new ones. 28 objects were listed in the NED as galaxies or objects from various catalogs with known redshifts and some of them already had information on emission lines in earlier publications. All objects were included in our observing program in order to improve spectral information. Comparison of our velocities with those of galaxies with already known redshift shows acceptable consistency within the uncertainties given.

Another 40 candidates observed were taken from the list of second priority candidates. As described in Paper IV two samples were created from this list. A "random selected sample" containing randomly selected objects from the list and the "APM selected sample" which uses additional information for selection. The "random selected sample" was created to access the fraction of BCGs in the second priority list and contains 43 objects. For 26 of them the spectral data were presented in Paper IV, while for the remaining 17 candidates observed with the CAHA $2.2 \mathrm{~m}$ telescope, the spectral information is presented here. The results of the analysis for this sample were presented in Paper IV. We found that the second priority list contains at most $10 \%$ BCG/HII-galaxies.

The second, "APM selected" sample comprises second priority candidates which are classified as non-stellar on Palomar Sky Survey plates (PSS) in the APM database, and have blue color according to the APM color system $((B-R)<1.0)$. Here we give spectral data for $23 \mathrm{ob}-$ jects from this sample. Except one all are ELGs confirming the efficiency of this selection criterium to pick up the BCG/HII-galaxies from the second priority list (cf. Paper IV).

\subsection{Emission-line galaxies}

The observed emission line galaxies are listed in Table 3 containing the following information:

Column 1: The object's IAU-type name with the prefix HS.

Column 2: Right ascension for equinox B1950.

Column 3: Declination for equinox B1950. The coordinates were measured on direct plates of the HQS and are accurate to $\sim 2^{\prime \prime}$ (Hagen et al. 1995).

Column 4: Heliocentric velocity and its rms uncertainty in $\mathrm{km} \mathrm{s}^{-1}$.

Column 5: Apparent B-magnitude obtained by calibration of the digitized photoplates with photometric standard stars (Engels et al. 1994), having an rms accuracy of $\sim 0.5$ for objects fainter than $m_{B}=16^{\mathrm{m}} 0$ (Popescu et al. 1996). Since the algorithm to calibrate the objective prism spectra is optimized for point sources the brightnesses of extended galaxies are underestimated. 
The resulting systematic uncertainties are expected to be as large as 2 mag (Popescu et al. 1996). For about 20\% of our objects, $B$-magnitudes are unavailable at the moment. We present for them blue magnitudes obtained from the APM database. They are marked by a "+" before the value in the corresponding column. According to our estimate they are systematically brighter by 0 m 92 than the $B$ magnitudes obtained by calibration of the digitized photoplates (rms 1. 02). Objects referred to Popescu \& Hopp (2000) have precise $B$-magnitude in Vennik, et al. (2000). We do not list them here for the sake of homogeneity. The $B$-magnitude for HS $1213+3636$ A was determined by eye estimate as 17 . 5 and marked by a ":" as less confident. Column 6: Absolute B-magnitude, calculated from the apparent $B$-magnitude and the heliocentric velocity. The only exception is made for HS $1213+3636 \mathrm{~B}$, a superassociation in the nearby galaxy NGC 4214 for which the distance is known from stellar photometry (see comments below). No correction for galactic extinction is made because all objects are located at high galactic latitudes and the corrections are significantly smaller than the uncertainties of the magnitudes.

Column \%: Preliminary spectral classification type according to the spectral data presented in this article. BCG means a galaxy possessing a characteristic HiI-region spectrum with low enough luminosity $\left(M_{B} \geq-20^{\mathrm{m}}\right)$. SBN and DANS are galaxies of lower excitation with a corresponding position in line ratio diagrams, as discussed in Paper I. SBN are the brighter fraction of this type. Here we follow the notation of Salzer et al. (1989). Three objects were recognized as Seyfert galaxies. Two of them (HS $1317+4521 \mathrm{~B}$ and HS $1616+3627$ ) are Sy1 galaxies due to the presence of broad Balmer lines and of broad [FeII] emission. Our spectrum of HS $1616+3627$ has insufficient quality to show this, but independent spectroscopy data of Grupe et al. (1999) clearly classify this object as a narrow-line Sy1 galaxy. The third one (HS 1220+3845) is a narrow-line ELG, which is classified as a Sy2 galaxy in diagnostic diagrams. Typical spectra of low-ionisation nuclear emission-line regions (LINERs) are identified for 3 galaxies. SA stands for two probable super-associations in two dwarf spiral galaxies. Six ELGs are difficult to classify. They are coded as NON.

Column 8: One or more alternative names, according to the information from NED. References are given to the other sources of the redshift-spectral information indicating that a galaxy is an ELG.

The spectra of all emission-line galaxies are shown in Appendix A, which is available only in the electronic version of the journal.

The results of line flux measurements are given in Table 4. It contains the following information:

Column 1: The object's IAU-type name with the prefix HS. By asterisk we note the objects observed during non-photometric conditions.

Column 2: Observed flux (in $10^{-16} \mathrm{erg} \mathrm{s}^{-1} \mathrm{~cm}^{-2}$ ) of the $\mathrm{H} \beta$ line. For several objects without $\mathrm{H} \beta$ emission line the fluxes are given for $\mathrm{H} \alpha$ and marked by a "+".
Columns 3-5: The observed flux ratios $[\mathrm{OII}] / \mathrm{H} \beta$, [OIII] $/ \mathrm{H} \beta$ and $\mathrm{H} \alpha / \mathrm{H} \beta$.

Columns 6, 7: The observed flux ratios [NII] $\lambda 6583 \AA / \mathrm{H} \alpha$, and $([\mathrm{SII}] \lambda 6716 \AA+\lambda 6731 \AA) / \mathrm{H} \alpha$.

Columns 8-10: Equivalent widths of the lines [OII] $\lambda 3727 \AA, \mathrm{H} \beta$ and [OIII] $\lambda 5007 \AA$. For several objects without detected $\mathrm{H} \beta$ emission line the equivalent widths are given for $\mathrm{H} \alpha$ and marked by a "+".

Below we give comments on some specific cases:

$H S 0948+3723$ and $H S 1430+4040$ : $\mathrm{H} \alpha$-emission in these galaxies is affected by cosmic ray hits. The intensity ratio of $\mathrm{H} \gamma / \mathrm{H} \beta$ is close to the recombination one with $c_{\mathrm{H} \beta}=0$. So we accepted for them the recombination $\mathrm{H} \alpha / \mathrm{H} \beta$ flux ratio, and corrected respectively the ratios of $[\mathrm{NII}] / \mathrm{H} \alpha$ and [SII] $/ \mathrm{H} \alpha$ in Table 4.

HS $1213+3636 A$ : this is a compact slightly elongated $\left(\sim 9^{\prime \prime}\right)$ object at $\sim 60^{\prime \prime}$ to NW from the center of the starbursting barred Magellanic type galaxy (SBm) NGC 4214 (with $M_{B}=-17^{\mathrm{m}} 0$ ). Its radial velocity of $522 \pm 35 \mathrm{~km} \mathrm{~s}^{-1}$ is higher by $\sim 220 \mathrm{~km} \mathrm{~s}^{-1}$ than the systemic velocity of NGC 4214 and higher by $\sim 260 \mathrm{~km} \mathrm{~s}^{-1}$ than the velocity of HI gas in this place (McIntyre 1997). This implies that this compact emission region is a kinematically detached object, either a background dwarf, or more probably, a tiny "satellite" galaxy, passing by its much more massive neighbouring galaxy. For the former case its distance from the Hubble flow is $7 \mathrm{Mpc}, M_{B}=-11^{\mathrm{m}} 7$ : and the size $\sim 0.3 \mathrm{kpc}$. For the latter case, if HS $1213+3636 \mathrm{~A}$ is located at the same distance as NGC 4214 (2.64 Mpc, as found through stellar photometry with HST by Drozdovsky et al. $2001)$, its $M_{B}=-9.6$ : and the size $\sim 0.1 \mathrm{kpc}$. The nature of this tiny galaxy can be checked in principle by the use of well known luminosity-abundance relationship for gas-rich galaxies. If it is situated far from NGC 4214 one should expect that this galaxy should have metallicity $Z \leq 1 / 30 Z_{\odot}$. On the other hand, if HS $1213+3636 \mathrm{~A}$ is connected to NGC 4214 , one can speculate that this companion formed of gas pulled out of the parent SBm galaxy as a result of its strong interaction with other galaxy. In this case the metallicity of this companion should be close to that of NGC 4214 , that is known to be $\sim 1 / 4-1 / 5 Z_{\odot}$ (e.g., Kobulnitsky \& Skillman 1996). Thus, metallicity determination of this dwarf with strong starburst will probably resolve the dilemma.

$H S 1213+3636 B$ : this well elongated object $\left(\sim 12^{\prime \prime}\right)$ is projected onto the middle part $\left(\sim 46^{\prime \prime}\right.$, or $\sim 0.6 \mathrm{kpc}$ to NWW from the center) of the same galaxy NGC 4214. It is at $\sim 25^{\prime \prime}$ to the South from HS $1213+3636$ A. Our velocity differs by only $\sim 60 \mathrm{~km} \mathrm{~s}^{-1}$ from the NGC 4214 systemic velocity and is well consistent with the HI velocity of $260 \mathrm{~km} \mathrm{~s}^{-1}$ at this place from McIntyre (1997). From our data it follows that HS $1213+3636 \mathrm{~B}$ is a super-association (SA) in a spiral arm of NGC 4214 . Its $M_{B}=-9$ m 8 : and the size $\sim 0.15 \mathrm{kpc}$. The spectra of both $\mathrm{A}$ and $\mathrm{B}$ objects were acquired in one slit, so their large velocity difference is out of doubt. 
HS 1311+3628: this elongated object $\left(\sim 11^{\prime \prime}\right)$ is projected onto the southern edge $\left(\sim 50^{\prime \prime}\right.$ to the South from the center) of Im galaxy UGC 8303 (Holmberg VIII). A large difference of its radial velocity $\left(1094 \pm 12 \mathrm{~km} \mathrm{~s}^{-1}\right)$ with that of the systemic velocity of the dwarf irregular UGC $8303\left(944 \pm 5 \mathrm{kms}^{-1}\right.$ from Huchra et al. 1995) and the local $\mathrm{HI}$ velocity in this region $\left(\sim 945 \mathrm{~km} \mathrm{~s}^{-1}\right)$ from the Hi map by Thean et al. (1997) (where this galaxy by error is called UGC 8314), implies that this object probably does not belongs to UGC 8303 and is a nearby separate star-forming dwarf. We observed this object twice on different nights and with different setups. Both spectra have the same redshifts within the observational uncertainties. HS $1311+3628$ is therefore not an HiI-region in UGC 8303 as suggested by Popescu et al. (1996).

HS 1311+3924: this is the irregular (probably merging) galaxy UGC 8315 and member of a group, with a radial velocity of $1215 \mathrm{~km} \mathrm{~s}^{-1}$ according to Garcia et al. (1993). We got the spectrum of only one of two bright knots in the galaxy elongated body, namely the NE one. While its velocity $1401 \pm 43$ differs by $186 \mathrm{~km} \mathrm{~s}^{-1}$ from that given by Garcia et al. (1993) the combined uncertainty of latter value is not well constrained. A long-slit spectrum along the major axis will be helpful for understanding the nature of this object.

$H S$ 1423+3945: the radial velocity of this object is very close to that of UGC 9242 (our $1480 \pm 35$ vs. $1440 \pm 11 \mathrm{~km} \mathrm{~s}^{-1}$ in the RC3 catalog) and therefore the object is very probably an SA in this edge-on dwarf spiral.

HS 1242+4058A: The nearby BCG HS 1242+4058B (cf. Paper II) is separated by $\sim 4^{\prime}$ from HS $1242+4058 \mathrm{~A}$ and the radial velocities differ by $\approx 300 \mathrm{kms}^{-1}$. They are probably not associated with each other.

$H S 1317+4521 B$ : The nearby BCG-candidate $\left(\sim 10^{\prime \prime}\right.$ to SW) HS $1317+4521 \mathrm{~A}$ is not observed yet.

$H S$ 1646+4003B: its faint companion $\left(\sim 30^{\prime \prime}\right.$ to SSW), HS $1646+4003 \mathrm{~A}$ was earlier identified as NON (cf. Paper II).

$H S \quad 0935+4135, \quad H S \quad 0958+3654, \quad H S \quad 1025+3707 B$, $H S 1027+4728, H S \quad 1206+4012, H S 1236+4532$, $H S 1433+4245, H S 1514+4602:$ In all these galaxies the only emission line suitable for redshift measurement is $\mathrm{H} \alpha$. A conservative estimate of their radial velocity uncertainty is $300 \mathrm{~km} \mathrm{~s}^{-1}$.

For some of the galaxies either the snap-shot spectra had strong enough emission lines and a well detected [OIII] $\lambda 4363 \AA$ or they were reobserved later with better $S / N$ ratio. For these galaxies we determined metallicities and the following galaxies appeared to have $\mathrm{O} / \mathrm{H} \leq 1 / 10(\mathrm{O} / \mathrm{H})_{\odot}$ : HS 1313+4521, HS 1330+3651, HS 1334+3957, HS $1408+4227$, HS $1417+4433$, HS $1545+4055$, HS $1650+3706$, HS $1655+3845$.

For two BCGs (HS $0940+4052$ and HS $1311+3628$ ) the blue WR-bump is more or less evident from the snap-shot spectra, and for 8 more BCGs this feature is probably present.
Several "BCG?" were listed in Table 3 with absolute magnitudes brighter than $-20{ }^{\mathrm{m}} 0$. In fact, these $M_{B}$ are uncertain, since they come from the estimates from the Hamburg Quasar Survey plates. Independent CCD measurements or estimates from alternative sources show that usually they are fainter by $0^{\mathrm{m}} 5-1^{\mathrm{m}} 0$, and should fulfill therefore the criterion $M_{B} \geq-20$. 0 (Kniazev et al. 2001b).

\subsection{Quasars}

The main criteria applied to search for BCGs are a blue continuum near $\lambda 4000 \AA$ and a strong emission line expected is [OIII] $\lambda 5007 \AA$ - in the wavelength region between $5000 \AA$ and the sensitivity break of the Kodak IIIa-J photoemulsion near $5400 \AA$ (see Paper I). For this reason faint QSOs with $\operatorname{Ly} \alpha \lambda 1216 \AA$ redshifted to $z \sim 3$ or with MgII $\lambda 2798 \AA$ redshifted to $\mathrm{z} \sim 0.8$ could be selected as BCG candidates. In Papers I-IV we already reported on the discovery of a number of such faint QSOs. They have been missed by the proper Hamburg Quasar Survey since the latter is restricted to bright QSOs $(B \leq 17-17.5)$. Here we report on the discovery of five high-redshift faint $(B>17.5)$ QSOs. For all but one of them, we identified Ly $\alpha \lambda 1216 \AA$ redshifted to $z \sim 3$ as the line responsible for its selection. One object (HS 1301+4233) shows a broad emission line tentatively identified with $\operatorname{MgII} \lambda 2798 \AA$. However this identification remains somewhat uncertain due to low signal-to-noise of the spectrum. Two of our new QSOs: HS $1224+4410$ and HS $1541+4452$, have recently appeared in the NED as radio sources with no redshift determination (reported as [HVG99] R17 in Hunter et al. 1999, and B3 1541+448A in Douglas et al. 1996). The data for all five quasars are presented in Table 5. Finding charts and plots of their spectra can be found on the www-site of the Hamburg Quasar Survey (http://www.hs.uni-hamburg.de/hqs.html).

\subsection{Non-emission-line objects}

In total, for 43 candidates no (trustworthy) emission lines were detected. We divided them into three categories.

\subsubsection{Absorption-line galaxies}

For four non-ELG galaxies the signal-to-noise ratio of our spectra was sufficient to detect absorption lines, allowing the determination of redshifts. The data are presented in Table 6 .

\subsubsection{Stellar objects}

To separate the stars among the objects missing detectable emission lines we cross-correlated a list of the most common stellar features with the observed spectra. In total, 21 objects with definite stellar spectra and redshifts close to zero were identified. All of them were 
crudely classified in categories from definite A-stars to Gstars, with most of them intermediate between $\mathrm{A}$ and $\mathrm{F}$. The data for these stars are presented in Table 7.

\subsubsection{Non-classified objects}

There was no possibility of classifying 18 objects without emission lines. Their spectra have too low signal-tonoise ratio to detect trustworthy absorption features, or the $E W \mathrm{~s}$ of their emission lines are too small.

\section{Discussion}

\subsection{The fifth list}

As result we have 209 observed candidates preselected on HQS objective prism plates, of which 169 were first priority candidates and 40 were second priority ones. 166 objects ( $79 \%$ from 209 objects) are found to be either ELGs (161), or quasars (5).

Of 161 ELGs 98 galaxies (61\%) were classified based on the character of their spectra and their luminosity as HiI/BCGs or probable BCGs.

Two of them (HS $1213+3636 \mathrm{~A}$ and HS $1311+3628$ ) are low-mass neighbours/satellites of the dwarf spiral NGC 4214 and of the Im galaxy UGC 8303. As the discussion of the local environment of BCGs is out of the scope of this paper, we refer to a recent analysis of this issue by Pustilnik et al. (2001).

HS $1213+3636 \mathrm{~B}$ and HS $1423+3945$ are SAs in the dwarf spirals NGC 4214 and UGC 9242.

Six ELGs are difficult to classify at all due to their poor signal-to-noise spectra. Six more ELGs were classified as Active Galactic Nuclei (AGNs): 3 as Seyfert galaxies and 3 as LINERs. The remaining 49 ELGs are objects with low excitation: either starburst nuclei galaxies (SBN and probable SBN) or their lower mass analogs - dwarf amorphous nuclear starburst galaxies (DANS or probable DANS).

By keeping a high fraction of BCGs ( $62 \%$ among the first priority candidates) we continue to have a high efficiency of discovery new BCGs, which is the main goal of the HSS. Since the completeness of the BCG sample under construction is an important parameter for many follow-up statistical studies, we observed a randomly selected sample of candidates from our list of second priority candidates. As discussed already in Paper IV at most 10\% of them turned out to be BCGs. Using additional information from the APM is an efficient means to pick up these BCGs among the second priority candidates: among 23 objects observed 10 BCGs (43\%) were discovered (Table 1).

\subsection{Summary of the present status of the survey}

Summarizing the results of the Hamburg/SAO survey presented in Papers I through V, we discovered altogether from the 1st priority candidates 433 new emission-line objects (25 of them are QSOs), and for 85 known ELGs we got quantitative data for their emission lines. At the moment the total number of confident or probable blue compact/low-mass HiI-galaxies reaches 360. Relative to all observed 493 ELGs the fraction of BCGs (360/493 or $73 \%$ ) demonstrates the high efficiency of the survey to find this type of galaxies. 21 more new BCGs and 20 other type ELGs are found among the second priority candidates. To estimate the total number of BCGs in the HSS zone we should count new BCGs expected from the remaining candidates and those selected in the HSS, but not observed by us since they already were known from other surveys. Thus we expect the total number of BCGs in this sky region to be $\sim 500$. This will be the largest homogeneous BCG sample in both hemispheres.

\section{Conclusions}

We made follow-up spectroscopy of the fifth list of candidates from the Hamburg/SAO Survey for ELGs. Summarizing the results of the spectroscopy, the analysis of spectral information and the discussion above we draw the following conclusions:

- The intended methods to detect ELG candidates on the plates of the Hamburg Quasar Survey give a reasonably high detection rate of emission-line objects. In total, out of both priority categories, we observed 209 objects among which we found 166 emission-line objects corresponding to a detection rate of $\sim 79 \%$.

- Besides the ELGs we found also 5 new quasars, mostly with Ly $\alpha$ in the wavelength region 4950-5100 $(z \sim 3)$, near the red boundary of the IIIa-J photoplates.

- The high fraction of BCG/Hir galaxies among all observed ELGs (about $61 \%$ in this paper) is in line with our main goal - to pick up efficiently a statistically well selected and deep BCG sample in the sky region under analysis.

Acknowledgements. This work was supported by the grant of the Deutsche Forschungsgemeinschaft No. 436 RUS 17/77/94. U.A.V. is very grateful to the staff of the Hamburg Observatory for their hospitality and kind assistance. Support by the INTAS grant No. 96-0500 was crucial to proceed with the Hamburg/SAO survey declination band centered on $+37.5^{\circ}$. SAO authors acknowledge also partial support from the INTAS grant No. 97-0033. We note that the use of APM facility was extremely valuable for selection methodology of additional candidates to BCGs from the 2nd priority list. This research has made use of the NASA/IPAC Extragalactic Database (NED) which is operated by the Jet Propulsion Laboratory, California Institute of Technology, under contract with the National Aeronautics and Space Administration. We have also used the Digitized Sky Survey, produced at the Space Telescope Science Institute under government grant NAG W-2166.

\section{References}

Bohlin, R. C. 1996, AJ, 111, 1743

Douglas, J. N., Bash, F. N., Bozyan, F. A., Torrence, G. W., \& Wolfe, C. 1996, AJ, 111, 1945 
Drozdovsky, I., et al. 2001, in Bad Honnef conference Dwarf galaxies and their environment, Jan. 23-27, 2001

Engels, D., Cordis, L., \& Köhler, T. 1994, Proc. IAU Symp. 161, ed. H. T. MacGillivray (Kluwer: Dordrecht), 317

Garcia, A. M., Bottinelli, L., Garnier, R., Gouguenheim, L., \& Paturel, G. 1993, A\&AS, 97, 801

Grupe, D., Beuermann, K., Mannheim, K., \& Thomas H.-C. 1999, A\&A, 350, 895

Hagen, H.-J., Groote, D., Engels, D., \& Reimers, D. 1995, A\&AS, 111, 195

Hopp, U., Engels, D., Green, R., et al. 2000, A\&AS, 142, 417, Paper III

Huchra, J. P., Geller, M. J., \& Corwin, H. G. Jr. 1995, ApJS, 99, 391

Hunter, D., van Woerden, H., \& Gallagher, J. 1999, AJ, 118, 2184

Kniazev, A. Y., Engels, D., Pustilnik, S. A., et al. 2001a, A\&A, 366, 771, Paper IV

Kniazev, A. Y., Pustilnik, S. A., Ugryumov, A.V., et al. 2001b, in preparation

Kobulnicky, H. A., \& Skillman, E. D. 1996, ApJ, 471, 211

Markarian, B. E., Lipovetsky, V. A., \& Stepanian, J. A. 1983, Afz, 19, 29

McIntyre, V. J. 1997, Publ. Astron. Soc. Australia, 14, 122
Oke, J. B. 1990, AJ, 99, 1621

Pesch, P., Stephenson, C. B., \& MacConnell, D. J. 1995, ApJS, 98,41

Popescu, C. C., \& Hopp, U. 2000, A\&AS, 142, 247

Popescu, C. C., Hopp, U., Hagen, H.-J., \& Elsässer, H. 1996, A\&AS, 116, 43

Pustilnik, S. A., Engels, D., Ugryumov, A. V., et al. 1999, A\&AS, 135, 299, Paper II

Pustilnik, S. A., Kniazev, A. Y., Lipovetsky, V. A., \& Ugryumov, A. V. 2001, A\&A, 373, 24

Salzer, J. J., MacAlpine, G. M., \& Boroson, T. A. 1989, ApJS, 70,479

Salzer, J. J., Moody, J. W., Rosenberg, J. L., Gregory, S. A., \& Newberry, M. V. 1995, AJ, 109, 2376

Stepanian, J. A. 1994, Proc. IAU Symp. 161, ed. H. T. MacGillivray (Kluwer: Dordrecht), 731

Thean, A. H. C., Mundell, C. G., Pedlar, A., \& Nicholson, R. A. 1997, MNRAS, 290, 15

Ugryumov, A. V., Pustilnik, S. A., Lipovetsky, V. A., Izotov, Yu. I., \& Richter, G. M. 1998, A\&AS, 131, 295

Ugryumov, A. V., Engels, D., Lipovetsky, V. A., et al. 1999, A\&AS, 135, 511, Paper I

Vennik, J., Hopp, U., \& Popescu, C. C. 2000, A\&AS, 142, 399 